\title{
Hybrid bi-dimensional empirical mode decomposition based enhancement technique for extreme low contrast UAV thermal images
}

\author{
R THILLAINAYAGI*(D) and K SENTHIL KUMAR \\ Department of Aerospace Engineering, Madras Institute of Technology, Anna University, \\ Chennai 600044, India \\ e-mail: thillaimit@gmail.com
}

MS received 20 April 2018; revised 28 March 2019; accepted 8 April 2019; published online 22 May 2019

\begin{abstract}
The performance of automatic target detection and classification systems are typically affected by reduced contrast quality introduced by external interferences. In particular for Unmanned Aerial Vehicle (UAV) captured thermal surveillance images, the effect is more evident. This advises the use of contrast enhancement technique as a solution to enhance the reduced contrast of hot regions for efficient target detection. In this paper, a simple and novel enhancement technique based on singular value decomposition (SVD) using Bi-Dimensional Empirical Mode Decomposition (BEMD) is proposed to enhance the hot regions in extreme low contrast thermal images captured by UAV. In the first step, the technique decomposes the thermal image into Intrinsic Mode Functions (IMFs) and residue by using BEMD. In the second step, it applies Contrast Limited Adaptive Histogram Equalization (CLAHE) in the residue for local contrast enhancement and then calculates the singular value matrix. In the third step, residue component is rescaled for further improvement of hot regions using scaling factor. In the fourth step, a detail enhanced IMF components are generated using gray scale transformation. Finally, the contrast enhanced residue undergoes Inverse BEMD (IBEMD) together with the detailed enhanced IMFs for enhanced image generation. Experimental results demonstrate that the proposed technique effectively enhances the contrast and details in the image with less visual artefacts than other state-of-the-art techniques.
\end{abstract}

Keywords. Unmanned aerial vehicle surveillance image; thermal image enhancement; bi-dimensional empirical mode decomposition; contrast limited adaptive histogram equalization; singular value decomposition.

\section{Introduction}

In recent years, unmanned aerial vehicles (UAV) are realized as a powerful and cost effective solution for obtaining photogrammetric data of surveillance and reconnaissance region [1]. Effectiveness of UAV reconnaissance mission mainly relies on the quality of the captured images [2]. To acquire high quality images, UAV's are equipped with imaging payloads such as visual or thermal camera, which has a unique advantage of giving insight into environmental condition as well as auxiliary navigation.

Visual cameras are usually employed in UAVs to capture the images as it offers better interpretation with minimal cost. However, most visual images are subject to degradation under poor visibility, darkness and adverse weather conditions [3-5]. Target detection and classification under these situations are complicated, which can lead to faulty diagnosis of target region. This emerges the necessity of thermal cameras, which are applicable for both poor

*For correspondence visibility and darkness scenarios and can take advantage of the heat signature about the target.

Thermal camera is more significant in recognizing targets as it delivers better understanding of the scenarios in complex situations such as shadows, smoke, fog, darkness, camouflaged objects or background clutters. However, the thermal image of reconnaissance region captured in the above situations has relatively low contrast and less intensity background compared to targets with relatively higher intensity [6]. Furthermore, contrast of the image is diminished significantly by factors such as altitude between the camera and target [7], camera orientation [8] and aerosol turbulence of UAV [1], which reduces the visibility of the target details. This means that, the image has small intensity difference between the background and target, obstructing the detection and recognition of the targets. Thus, contrast enhancement technique is needed to improve the visibility between the target and background in the thermal images. The applications using thermal images such as border patrolling [9], search and track [10] and search and rescue for disaster relief management [11] necessitates thermal image enhancement as a basic pre- 
processing step to get the clear details about the potential intruders and survivors, so that an alert can be sent to the human-in-the-loop systems.

Existing techniques of thermal image enhancement focus only on the issues related to images captured by static camera, based on global and local histogram equalization techniques and no effort has been made to address the issues in UAV thermal images. Global histogram equalization (GHE) is a simple and widely used method, however, it suffers excessive contrast enhancement and fails to enhance the local details, which makes the resultant image unacceptable [12, 13]. Contrast limited adaptive histogram equalization (CLAHE) is a local histogram equalization technique proposed to overcome the limitation of GHE [14]. The CLAHE well enhances the local detail of the image but fails to limit the background noise.

Multi scale decomposition based image enhancement approaches have been proposed to overcome the limitations of local histogram equalization technique. Bilateral filter and dynamic range partitioning (BF\&DRP) has been proposed to improve the visual interpretation of thermal images [15]. The thermal image is initially separated into detail layer and base layer and then the two layers are enhanced using different approaches. Though it enhances the image well, it is subjected to severe gradient reversal artefacts and generates noise in the background region. Lidong et al [16] proposed a combined approach of discrete wavelet transform (DWT) and CLAHE, by applying different enhancement techniques on low and high frequency subbands to enrich the contrast of the image but it is not suitable for real time applications, as it requires empirical definition of various parameters making it computationally complex. Compared to wavelet transform, bi-dimensional empirical mode decomposition (BEMD) offers better frequency and temporal resolutions of images. Yang et al [17] proposed a combined approach of BEMD and principal component analysis (PCA) to enhance thermal images of rotating machine conditions. The thermal image is initially equalized by GHE and then is iteratively decomposed into intrinsic mode functions (IMFs). The obtained IMFs are expanded and fused with residue using PCA to produce the enhanced image. This method of enhancement is not suitable for images with extreme low contrast as the GHE utilized here, enhances the background rather than target.

In this paper, considering the features of UAV captured thermal images, in extreme low contrast, a novel enhancement approach using BEMD with SVD based illumination correction is proposed. The aim of this work is to enhance the contrast and emphasise edges of the image. Image enhancement using BEMD gives an opportunity for decomposing the image into different frequency component namely, IMFs (high frequency) and residue (low frequency). Therefore, different enhancement approach can be applied to enrich the high frequency (e.g., edge information) and low frequency component (e.g., luminance information) easily. In order to enhance the edge details, gray scale transformation is applied on the IMFs and to improve the contrast, SVD is applied on the residue component. Finally, the high contrast thermal image is obtained with better region enhancement and less structural damage.

This paper is structured as follows. Section 2 gives a global presentation of BEMD, CLAHE, SVD and mechanism of the proposed method for the enhancement of low contrast UAV thermal images in detail. Experimental results are validated in section 3 to verify the superiority of the proposed approach. Finally, concluding remarks of the proposed approach are given in section 4 .

\section{Materials and methods}

\subsection{Image database}

Test images were captured at various altitudes during poor visiblity and night environment by using Tau 2 and Hawkvision thermal imager mounted on hexa rotor type UAV to examine the region on the ground. The dimensions of the images are typically set to $320 \times 240$ and $640 \times 576$ pixels and transmitted to the portable ground control station and shared to the user for further processing. Contrast enhancement is carried out in offline mode for the images containing single or multiple targets.

\section{$2.2 B E M D$}

The BEMD is applied on the thermal image to obtain the IMFs and residue component. Consider the image $I(u, v)$, $u=1, \ldots M, u=1, \ldots N$, where $M$ and $N$ denote the total number of rows and columns in the image, respectively. The steps involved in the BEMD decomposition process are briefly summarized as follows [17]:

1. Process initialization: $r_{0}(u, v)=I(u, v)$ (the residue) and $i=1$ (index number of IMF).

2. Extraction of the $i^{\text {th }} I M F$ :

i. Initialize $h_{0}(u, v)=r_{i-1}(u, v), j=1$

ii. Find all the local maxima and minima of $h_{j-1}(u, v)$.

iii. Do surface interpolation using the maxima and minima points from step (ii) to obtain a maximum envelope $U_{j-1}(u, v)$ and minimum envelope $L_{j-1}(u, v)$.

iv. Compute the average of the maximum and the minimum envelopes:

$$
A_{j-1}(u, v)=\left[U_{j-1}(u, v)+L_{j-1}(u, v)\right] / 2
$$

v. Update

$$
h_{j}(u, v)=h_{j-1}(u, v)-A_{j-1}(u, v)
$$

and $j=j+1$ 
vi. Calculate Stopping criterion (SD) for decomposition process as follows

$$
S D_{i j}=\sum_{i=1}^{M} \sum_{j=1}^{N} \frac{\left|h_{i(j-1)}(u, v)-h_{i j}(u, v)\right|^{2}}{h_{i(j-1)}^{2}(u, v)}
$$

Reiterate steps (ii) to (vi) until $S D_{i j} \leq \xi$, where $\xi$ is an a priori constant, then $s_{i}(u, v)=h_{j}(u, v)$ is $\mathrm{i}^{\text {th }}$ IMF.

3. Once the IMF is obtained, update the residue $r_{i}(u, v)=r_{i-1}(u, v)-s_{i}(u, v)$

4. Reiterate steps (2)-(3) with $i=i+1$ until the number of extrema in $r_{i}(u, v)$ is less than 2 .

The generated IMFs and residue have the same size as the original image and it can be reconstructed without any loss of information using Eq. (4)

$$
I(u, v)=\sum_{K=1}^{l} I M F_{K}(u, v)+R(u, v)
$$

where $R(u, v)$ denotes the final residue, $l$ is the number of IMFs, $\operatorname{IMF}_{K}(u, v)$ is the $\mathrm{K}^{\text {th }} \mathrm{IMF}$.

The edge information of the image is bounded in IMFs and the luminance information is bounded in residue. In this paper, IMFs have been considered for edge enhancement and residue has been considered for contrast enhancement.

In this work, BEMD is applied on the low contrast image to decompose it into three IMFs and one residue component, which is found to be more suitable to enhance the contrast. For gray scale transformation of IMFs component, the parameter $k$ is set to 2 .

\subsection{CLAHE}

CLAHE is a local contrast enhancement technique, which can be applied to residue component of input thermal image to improve the local details effectively. The key idea behind this technique is to split the image into non overlapping blocks and equalize each by using clipping threshold and spread the pixels equally to all gray levels $[14,18]$. Further, a bilinear interpolation technique is used to limit discontinuity issues at the non overlapping block boundaries, which is generated during the mapping process [16].

\section{$2.4 S V D$}

SVD has been employed for face recognition [19], water marking [20] and also for the enhancement of low contrast images [21, 22]. SVD is used to decompose the rectangular or square image matrix $(I)$ into the product of three matrices.

$$
I=U S V^{T}
$$

where $U$ and $V$ are orthogonal square matrices and $S$ is matrix which contains singular values of matrix $I$ on its main diagonal and $T$ is a transpose operator. Elements of $U$ and $V$ known as left and right singular vectors of $I$. Singular vectors indicate inherent structural and geometry properties of the image, whereas singular values indicate the luminance properties of the image. Any amendment on singular values will directly influence the intensity information of the image without affecting the structural content.

\subsection{Proposed method}

The proposed approach of contrast enhancement for thermal image frames taken by UAV is done in five steps. The first step is to decompose the low contrast image into IMFs and residue using BEMD as shown in figure 1. The second step is to apply CLAHE on the residue for enhancing local contrast. The third step is to find the scaling factor for enriching the contrast of hot regions in the residue using SVD. The fourth step is to apply gray scale transformation on the IMFs for enhancing edge details and the final step is to reconstruct the enhanced thermal infrared image frame by adding the processed IMFs and with residue together. The work flow diagram of the proposed enhancement scheme is shown in figure 2 .

2.5a Contrast enhancement of residue component: In the proposed method, low contrast thermal image frame is applied as an input to the BEMD block for decomposing it into $\mathrm{IMF}_{1}, \mathrm{IMF}_{2}, \mathrm{IMF}_{3}$ and residue components, where the residue component encloses luminance information and the IMF components encloses edge information. The CLAHE is then applied on residue component to improve the local contrast, which preserves the edge information of IMFs from possible degradation.

SVD is applied on the residue component of CLAHE enhanced and direct BEMD to generate the values of $U, S$ and $V$ in the following equations:

$$
\begin{aligned}
& R_{C}=U_{R_{C}} S_{R_{C}} V_{R_{C}} \\
& R_{D}=U_{R_{D}} S_{R_{D}} V_{R_{D}}
\end{aligned}
$$

where $R_{C}$ and $R_{D}$ are singular value matrices of CLAHE enhanced and direct BEMD, respectively. $U_{R_{C}}$ and $U_{R_{D}}$ are orthogonal matrices, $V_{R_{C}}$ and $V_{R_{D}}$ are orthogonal transpose matrices. $U$ and $V$ matrices contain structural information of the applied image. $S_{R_{C}}$ and $S_{R_{D}}$ are diagonal matrices which contain the luminance information of the applied image.

The maximum element in $S_{R_{C}}$ and $S_{R_{D}}$ are computed to determine the scaling factor for enhancing the luminance of hot regions is obtained by using 
$\mathrm{IMF}_{1}$

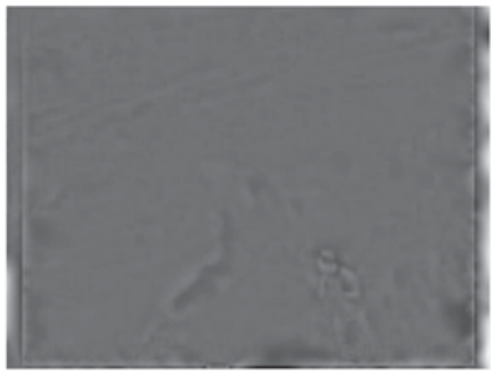

$\mathrm{IMF}_{3}$

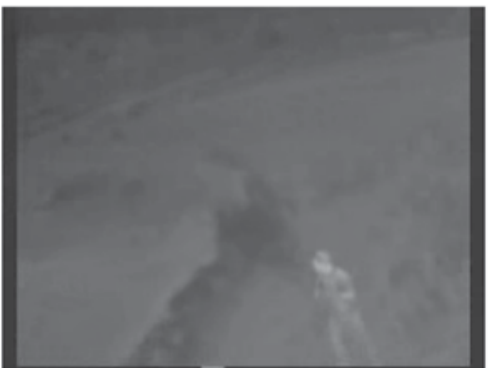

(a)

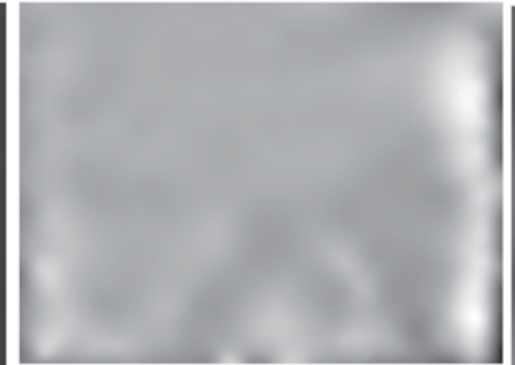

(b)
$\mathrm{IMF}_{2}$

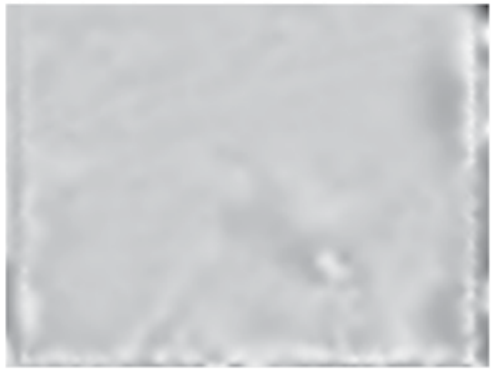

Residue

Figure 1. BEMD decomposition of an input image. (a) Original image, (b) $\mathrm{IMF}_{1}, \mathrm{IMF}_{2}, \mathrm{IMF}_{3}$ and Residue images.

$$
\zeta_{L}=\frac{\max S_{\mathrm{R}_{\mathrm{C}}}}{\max S_{R_{D}}}
$$

where $\zeta_{L}$ is the scaling factor for luminance.

The luminance enhanced singular value matrix and enhanced residue component are computed using the following equations

$$
\begin{gathered}
S^{\wedge}=\zeta S_{R_{D}} \\
R_{D}^{\wedge}=U_{R_{D}} S^{\wedge} V_{R_{D}}^{T}
\end{gathered}
$$

The enhanced residue component is reconstructed using the elements of direct BEMD to prevent the noise amplification issue of CLAHE.

2.5b Edge enhancement of IMF component: Before the reconstruction process, the IMF components are expanded to obtain detail enhancement, by multiplying factor $k(1<k<3)$.

2.5c Image Reconstruction: The enhanced thermal image is produced by recombining all the detail enhanced IMFs with the contrast enhanced residue through a reconstruction process using Eq. (4) and the produced result has well enhanced hot regions with sharper edges and fewer artefacts.

Figure 3 is an illustration for choosing the parameter $k$. Figure $3 \mathrm{a}$ is an original image, figure $3 \mathrm{~b}-\mathrm{e} €$ show the enhancement results of proposed method with $k$ equal to
1.5, 2.0, 2.5 and 3.0 respectively. As we can see from figures, the proposed method with $k=2.0$ yields highest subjective quality and the proposed method with $k=1.5$ cannot improve the contrast of the image with sufficient quality. The proposed method with $k=2.5$ and $k=3.0$ loses some details of the image because of over enhancement. Note that, for higher values of $k$, the high frequency part enhanced too much; therefore, the edges become too ridged. On the other hand, for lower values of $k$, the loss of edges is prominent.

In this work, a computationally simple and cost-effective method is proposed to enhance the images captured by UAV and is carried out in offline mode. The value of the parameter $k$ is tuned empirically for the images.

The work can be extended in future, to improve the efficiency of the proposed method by defining the parameter $k$ automatically for various images, making it more appropriate for online applications. This can be achieved by incorporating machine learning algorithms relying on training data with desired features. However, number of training data, size, network construction, layers selection which are involved in learning algorithms based on training data will increase the computational load and processing time.

Instead, the utilization of deep learning reinforcement algorithms to tune the parameter $k$ would fetch a fruitful result of reduced processing time compared to the machine learning algorithms based on training data with better performance. 


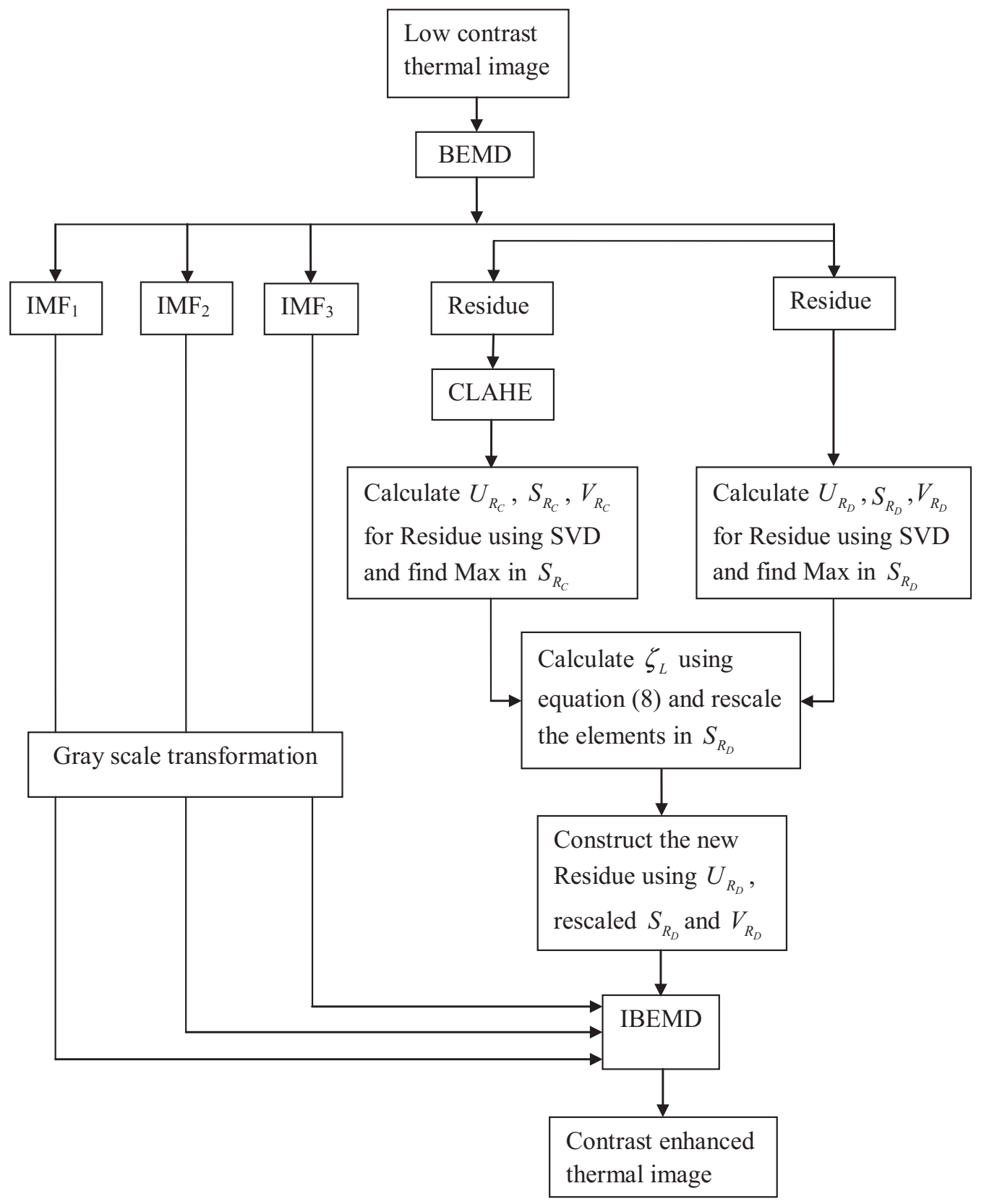

Figure 2. Work flow diagram of the proposed contrast enhancement technique.

Steps involved in tuning the parameter $k$ :

1. Extract some local features from an image and study the relation between these features and the optimal tuning parameter.

2. Define objective function to enhance the IMFs component of BEMD decomposed image.

3. Design parameter tuning policy network (based on convolutional neural network) for optimizing the value of parameter $k$ to enhance the IMFs components. Further, it should be designed in such a way that an intellectual observation of image features and characteristics would be made necessary to determine the magnitude of parameter $k$ while tuning the parameter $k$.

4. Train the network via deep learning reinforcement to get the optimum results.

5. Reconstruct the enhanced image using IBEMD with optimum visual quality.

The steps mentioned above to tune the parameter $k$ and reconstruction of enhanced image would have a considerable processing time. However, this can be reduced with the use of embedded processor during computation. 


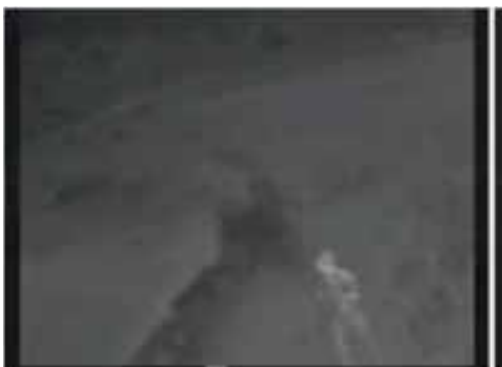

(a)

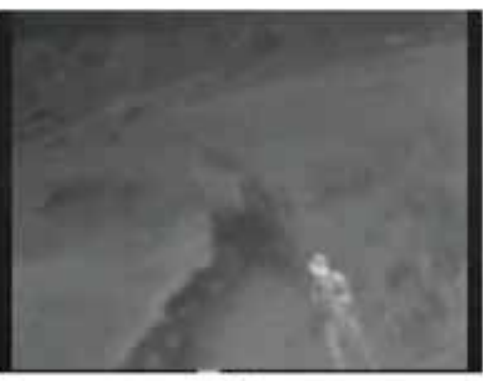

(b)

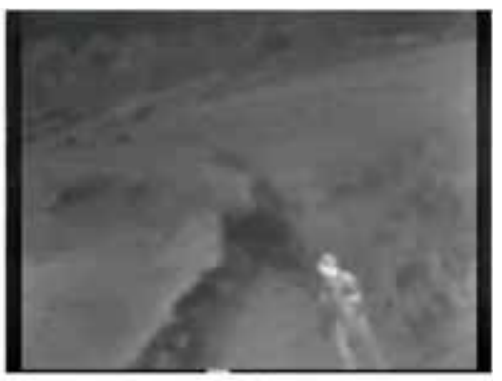

(c)

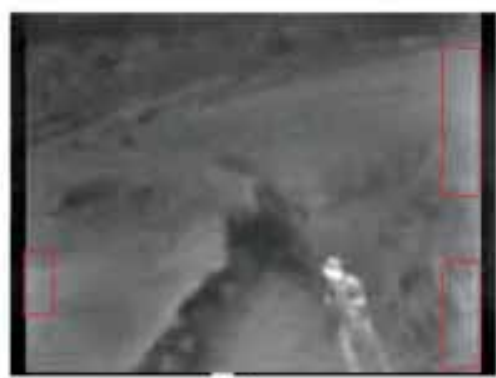

(d)

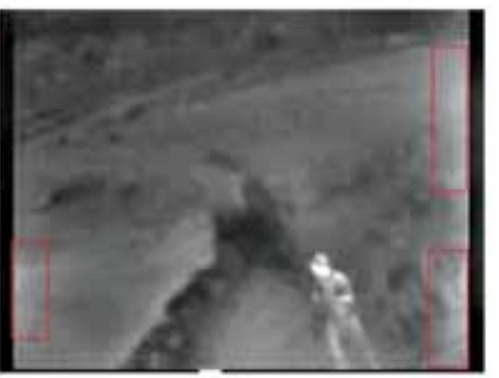

(e)

Figure 3. Illustration for the parameter $k$ choosing: (a) Original image, (b), (c), (d) and (e) are enhanced images of proposed method with parameter $k$ equals to $1.5,2.0,2.5$ and 3.0 , respectively.

\section{Results and discussion}

In this paper, numerous experiments are carried out to assess and compare the performance of the proposed technique with three different state-of-the-art techniques: GHE [12], DWT-CLAHE [16], and BEMD-PCAF [17]. Both qualitative results in the form of visual images and quantitative results are considered for performance assessment.

\subsection{Qualitative assessment}

For qualitative assessment, five low contrast thermal images are taken to validate the effectiveness of the proposed technique and are shown in figures 4, 5, 6, 7 and 8.

As shown in figures 4, 5, 6, 7 and 8, GHE not only enhances the hot region of interest, but also background region. The histogram of low contrast thermal images are mostly dark, GHE method makes artefacts. In this condition, the hot regions of interest are brightened more than necessary, which makes the image quality insufficient. DWT-CLAHE suffers under saturation of pixels and fails to discriminate the hot regions of interest from background. This can be clearly seen in figures 4, 5, 6, 7 and 8. BEMDPCAF method suffers over equalization and hence features are completely washed out in the obtained results. This is because the pixels of the original image are initially over equalized by GHE and the resultant pixels are further enhanced by gray scale transformation process of BEMD-
PCAF method. The three state-of-the-art techniques mentioned above do not produce better and visually satisfactory results. On the other hand, the proposed method obtains a better region enhancement and discriminates the hot region of interest from background with higher intensity. Application of CLAHE on residue and gray scale transformation on IMFs component avoids over enhancement and improves details of the enhanced image, respectively.

\subsection{Quantitative assessment}

For quantitative assessment of contrast enhancement methods, four parameters namely discrete entropy [23], measure of enhancement (EME) [24], Mean Structural Similarity Index Measurement (MSSIM) [25] and Feature Similarity Index Measurement (FSIM) [26] are considered in this paper.

The first assessment parameter considered here is discrete entropy, which indicates the degree of detail enhancement in an image and it is given as

$$
H=\sum_{i=0}^{L-1} p(i) \log _{2} p(i)
$$

where $p(i)$ represents the probability of intensity value $i$ appearing in the processed image.

The next assessment parameter considered here is EME, which measures the average contrast in an image and it is given as 


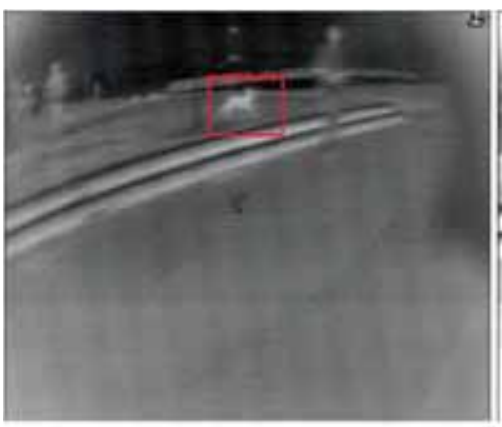

(a)

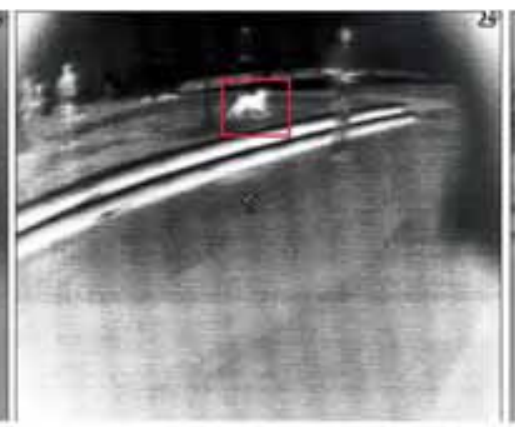

(b)

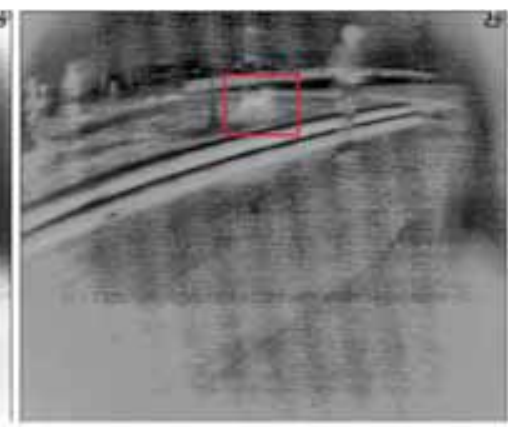

(c)

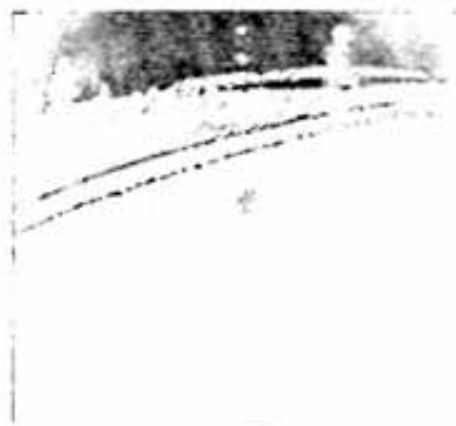

(d)

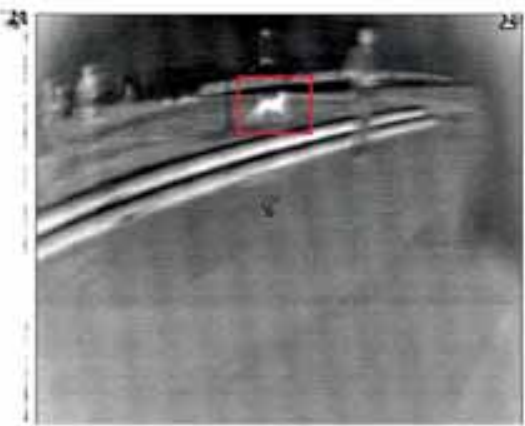

(e)

Figure 4. Contrast enhancement results of all methods tested on the dataset of image 1: (a) original image, (b) enhanced by GHE, (c) enhanced by DWT-CLAHE, (d) enhanced by BEMD-PCAF and (e) enhanced by the proposed method.

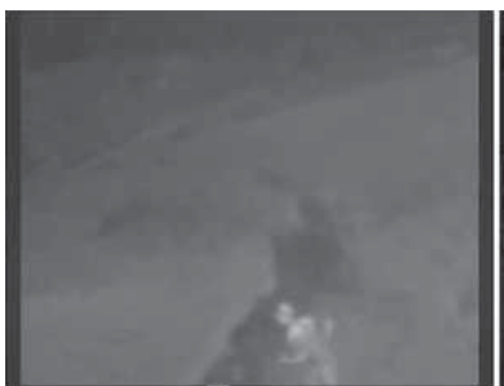

(a)

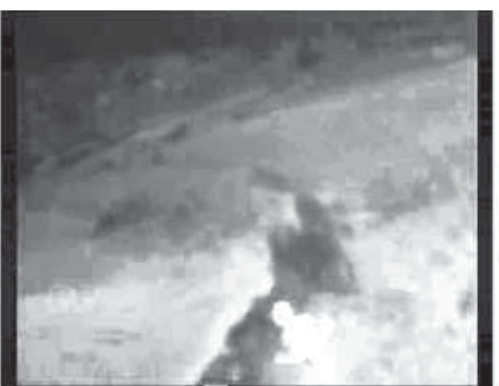

(b)

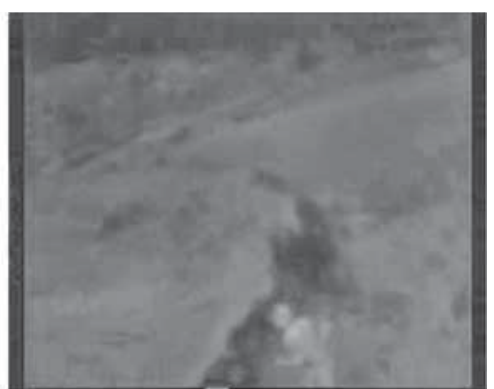

(c)

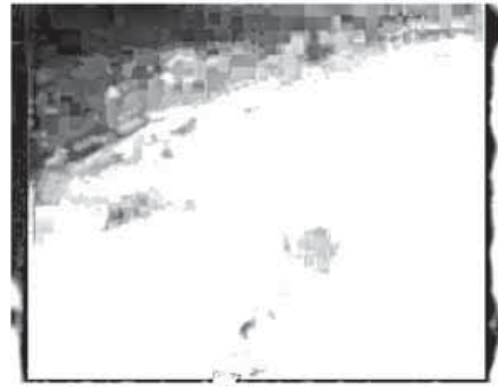

(d)

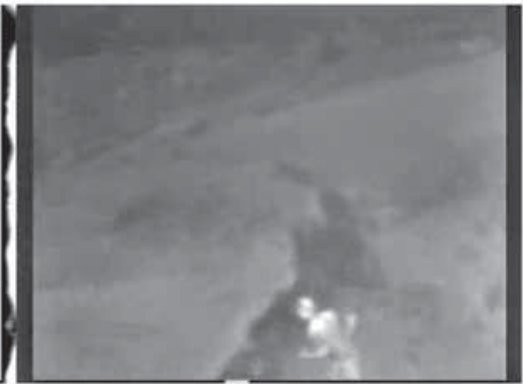

(e)

Figure 5. Contrast enhancement results of all methods tested on the dataset of image 2: (a) original image, (b) enhanced by GHE, (c) enhanced by DWT-CLAHE, (d) enhanced by BEMD-PCAF and (e) enhanced by the proposed method.

$$
E M E=\frac{1}{k_{1} k_{2}} \sum_{l=1}^{k_{2}} \sum_{k=1}^{k_{1}} 20 \log \left(\frac{\mathrm{I}_{\max }(\mathrm{k}, \mathrm{l})}{\mathrm{I}_{\min }(k, l)}\right)
$$

where, $I_{\max }$ and $I_{\min }$ are the maximum and minimum pixel intensity values in a block. A higher value of EME indicates better performance of contrast enhancement. 


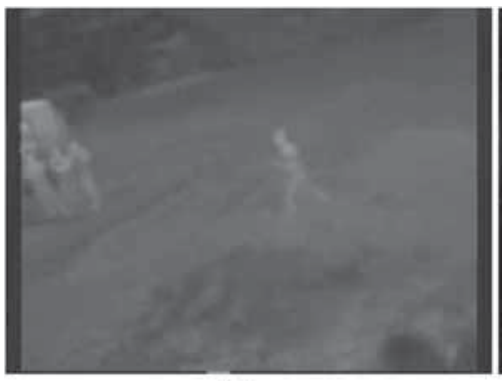

(a)

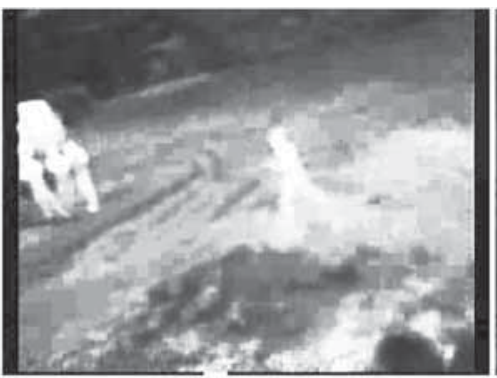

(b)

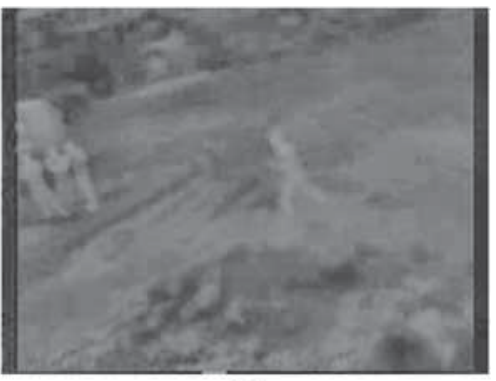

(c)

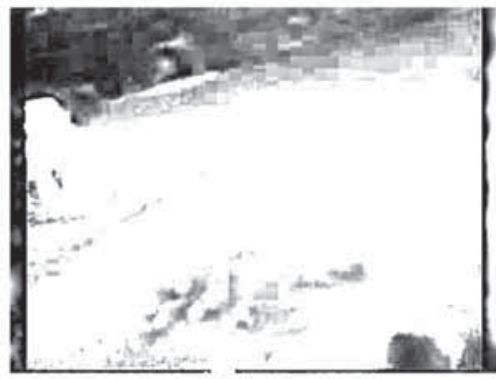

(d)

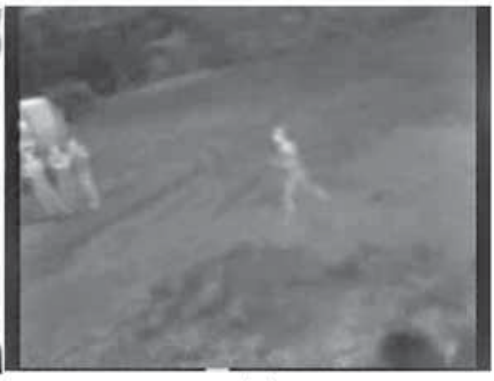

(e)

Figure 6. Contrast enhancement results of all methods tested on the dataset of image 3: (a) original image, (b) enhanced by GHE, (c) enhanced by DWT-CLAHE, (d) enhanced by BEMD-PCAF and (e) enhanced by the proposed method.

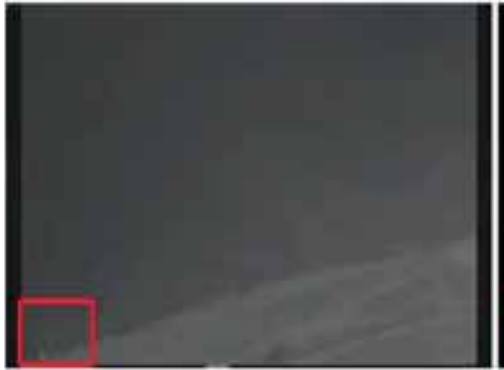

(a)

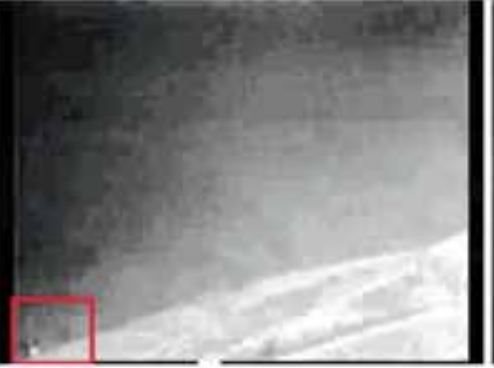

(b)

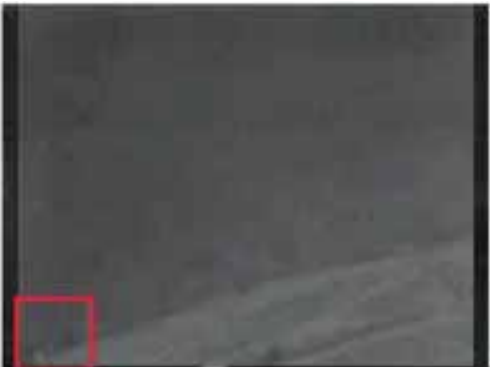

(c)

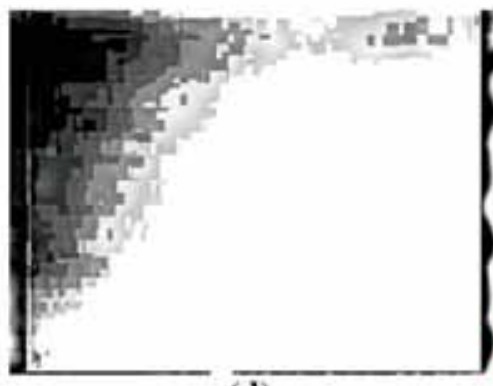

(d)

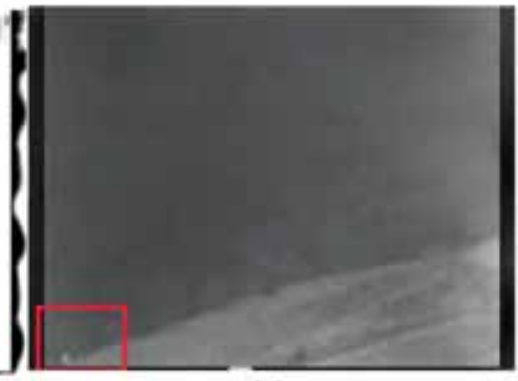

(e)

Figure 7. Contrast enhancement results of all methods tested on the dataset of image 4: (a) original image, (b) enhanced by GHE, (c) enhanced by DWT-CLAHE, (d) enhanced by BEMD-PCAF and (e) enhanced by the proposed method.

Another quality assessment parameter considered here is MSSIM, which calculates the degradation of structural information between the original and enhanced image and it is given as

$$
\operatorname{SSIM}(x, y)=\frac{\left(2 \mu_{x} \mu_{y}+C_{1}\right)\left(\left(2 \sigma_{x y}+C_{2}\right)\right)}{\left(\mu_{x}^{2}+\mu_{y}^{2}+C_{1}\right)\left(\sigma_{x}^{2}+\sigma_{y}^{2}+C_{2}\right)}
$$




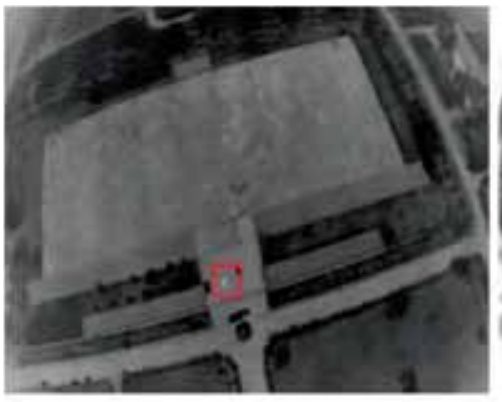

(a)

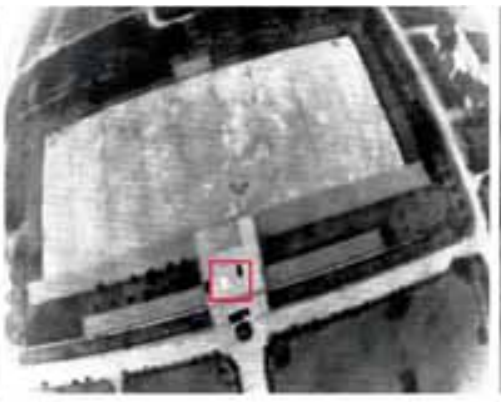

(b)

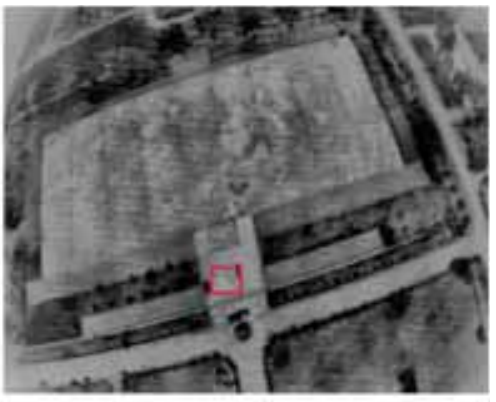

(c)

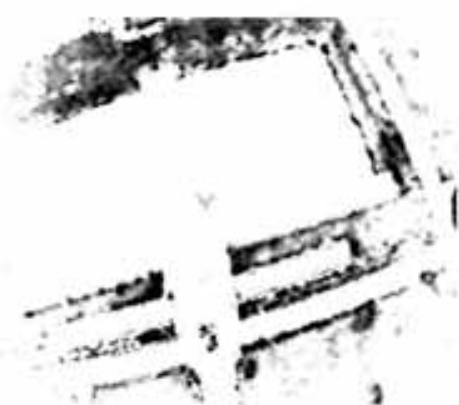

(d)

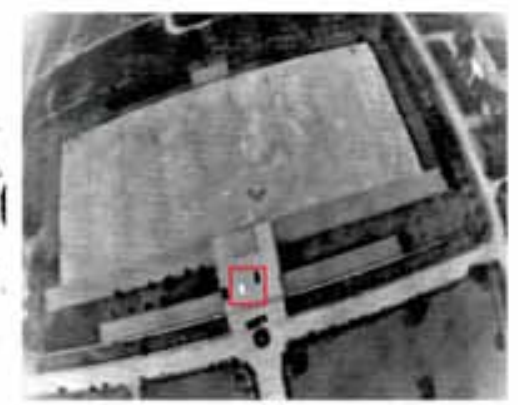

(e)

Figure 8. Contrast enhancement results of all methods tested on the dataset of image 5: (a) original image, (b) enhanced by GHE, (c) enhanced by DWT-CLAHE, (d) enhanced by BEMD-PCAF and (e) enhanced by the proposed method.

$$
\operatorname{MSSIM}=\frac{1}{M} \sum_{j=1}^{M} \operatorname{SSIM}\left(x_{j}, y_{j}\right)
$$

where, $\mathrm{M}$ is the number of local windows in the image, $\mu_{x}, \mu_{y}$ are the averages of $x$ and $y ; \sigma_{x}^{2}$ and $\sigma_{y}^{2}$ are the variance of $x$ and $y ; \sigma_{x y}$ is the covariance of $x$ and $y ; C_{1}$ and $C_{2}$ are the constants. A higher value of MSSIM represents a better visual similarity between the original and enhanced image.

The final assessment measure considered here is FSIM, which computes the local feature similarity between original and enhanced image and it is given as

$$
\operatorname{FSIM}_{o, e}=\frac{\sum_{x} F S_{o, e}[x] . P C_{o, e}[x]}{\sum_{x} P C_{o, e}[x]}
$$

where $F S_{o, e}[x]$ denotes the similarity between original and enhanced images and $P C_{o, e}[x]$ denotes the phase congruency of co-located block $x$ between original and enhanced images. A higher value of FSIM represents a better feature similarity between the original and enhanced image.

The quantitative results of various contrast enhancement methods are provided in tables 1, 2, 3 and 4. From the tabulated values it is observed that the discrete entropy parameter shows higher performance for the proposed method, than GHE, DWT-CLAHE and BEMD-PCAF. In images enhanced using DWT-CLAHE, and GHE, there is loss of some image features leading to relatively lower discrete entropy values. In images enhanced using BEMD-
PCAF, there is loss of complete image features due to wash out effect leading to lowest entropy values. Taking the above reasons into consideration, the proposed method can be concluded as robust in discovering the hidden image details.

Higher EME values are achieved for BEMD_PCAF, DWT-CLAHE and GHE compared to the proposed method. The existing methods constitute visually not suitable images; undesirable artefacts such as over equalization of pixels introduced in the enhanced images leading to sharp increase in image contrast and eventually increase the EME value.

In order to avoid the above mentioned misconceptions and for a reliable contrast assessment, MSSIM and FSIM metrics are taken into account. Comparisons of MSSIM and FSIM for the considered methods reveal that the proposed method achieves best structural and feature information than other methods. DWT-CLAHE, GHE and BEMDPCAF show decrease in performance. DWT-CLAHE suffers under saturation of pixels, which deteriorates the structural and feature information. The deterioration of features of hot regions observed in GHE reduces the MSSIM and FSIM value. The BEMD-PCAF uses GHE as initial equalization process, which further reduces MSSIM and FSIM value. Hence, the enhancement results obtained using the proposed method have highest structural similarity, feature similarity and natural look with improved visibility of image details. 
Table 1. Comparison of Discrete Entropy for various contrast enhancement methods.

\begin{tabular}{lcccc}
\hline Test images\# & GHE & DWT-CLAHE & BEMD-PCAF & Proposed method \\
\hline Methods & & & & \\
Image1 & 5.9686 & 7.0631 & 1.6904 & 7.4150 \\
Image2 & 5.5666 & 6.4572 & 3.3814 & 6.9349 \\
Image3 & 5.3119 & 6.4192 & 3.4752 & 6.7353 \\
Image4 & 5.1056 & 5.7806 & 3.3988 & 6.4497 \\
Image5 & 5.9638 & 7.2980 & 2.2716 & 7.7047 \\
Mean discrete entropy & 5.5833 & 6.6036 & 2.8434 & 7.0479 \\
\hline
\end{tabular}

Table 2. Comparison of EME for various contrast enhancement methods.

\begin{tabular}{lcccc}
\hline Test images\# & GHE & DWT-CLAHE & BEMD-PCAF & Proposed method \\
\hline Methods & & & & \\
Image1 & 7.7773 & 7.5236 & 12.0334 & 6.8256 \\
Image2 & 4.2488 & 4.5495 & 7.7466 & 3.3583 \\
Image3 & 6.6729 & 5.9523 & 10.9297 & 4.7049 \\
Image4 & 3.0450 & 2.4996 & 5.0754 & 2.1382 \\
Image5 & 9.2443 & 11.9982 & 11.1832 & 8.8811 \\
Mean EME & 6.1976 & 6.5046 & 9.3937 & 5.1816 \\
\hline
\end{tabular}

Table 3. Comparison of MSSIM for various contrast enhancement methods.

\begin{tabular}{lcccc}
\hline Test images\# & GHE & DWT-CLAHE & BEMD-PCAF & Proposed method \\
\hline Methods & & & & \\
Image1 & 0.7479 & 0.7612 & 0.0188 & 0.8794 \\
Image2 & 0.6558 & 0.8827 & 0.0947 & 0.9055 \\
Image3 & 0.5417 & 0.8337 & 0.0774 & 0.9011 \\
Image4 & 0.6587 & 0.9192 & 0.0852 & 0.9328 \\
Image5 & 0.7415 & 0.7738 & 0.0252 & 0.7854 \\
Mean MSSIM & 0.6691 & 0.8341 & 0.0602 & 0.8808 \\
\hline
\end{tabular}

Table 4. Comparison of FSIM for various contrast enhancement methods.

\begin{tabular}{lcccc}
\hline Test images\# & GHE & DWT-SVD & BEMD-PCAF & Proposed method \\
\hline Methods & & & & \\
Image1 & 0.8300 & 0.8189 & 0.0736 & 0.8918 \\
Image2 & 0.7880 & 0.9044 & 0.2443 & 0.9471 \\
Image3 & 0.6822 & 0.8640 & 0.1928 & 0.9320 \\
Image4 & 0.8326 & 0.9276 & 0.2921 & 0.9503 \\
Image5 & 0.8388 & 0.8365 & 0.2531 & 0.8689 \\
Mean FSIM & 0.7943 & 0.8703 & 0.2118 & 0.9180 \\
\hline
\end{tabular}

\section{Conclusions}

The authors have proposed a novel enhancement technique, that is, BEMD-CLAHE-SVD, which combine CLAHE and SVD with BEMD decomposition. This technique is dedicated for the enhancement of edges and contrast of the extremely low contrast thermal images captured by UAV.
Gray scale transformation is applied on the IMFs for enhancing edge details and CLAHE is applied on the residue for enhancing contrast of the image, respectively. The contrast of hot regions of interest in the residue is improved by the scaling factor. The performance of the proposed technique is compared with three well-known enhancement techniques and the experimental results reveal 
the superiority of the proposed technique over other techniques in terms of details enhancement, hot region enhancement and feature extraction effectively. Moreover, the proposed technique can be used as a key step for enhancing the targets clarity in the application of UAV based target detection and classification system, sensitive border management and search and rescue operations. In future, the work can be extended to improve the efficiency of the proposed method by automatically defining the enhancement parameter $k$ for various images, making it more appropriate for online applications.

\section{References}

[1] Kedzierski M and Wierzbicki D 2016 Methodology of improvement of radiometric quality of images acquired from low altitudes. Meas. 92: 70-78

[2] Huang Y, Ding W and Li H 2016 Haze removal for UAV reconnaissance images using layered scattering model. Chin. J. Aeronaut. 29: 502-511

[3] Choi Y, Kim N, Hwang S and Kweon I S 2016 Thermal image enhancement using convolutional neural network. In: Proceedings of the IEEE International Conference on Intelligent Robots and Systems (IROS), pp. 223-230

[4] Wu Y, Lim J and Yang M H 2013 Online object tracking: A benchmark. In: Proceedings of the IEEE International Conference on Computer vision and pattern recognition (CVPR), pp. 2411-2418

[5] Kumar S, Marks T K and Jones M 2014 Improving person tracking using an inexpensive thermal infrared sensor. In: Proceedings of the IEEE International Conference on Computer Vision and Pattern Recognition Workshops, pp. 217-224

[6] Ashiba H I, Awadalla K H, El-Halfawy S M and Abd ElSamie F E S 2008 Homomorphic enhancement of infrared images using the additive wavelet transform. Prog. Electromagn. Res. 1: 123-130

[7] Liu H, Wang W, He Z, Tong Q, Wang X, Yu W and Ly M 2015 Blind Image quality evaluation metrics design for UAV photographic application. In: Proceedings of the IEEE International Conference on Cyber Technology in Automation, Control, and Intelligent Systems (CYBER), pp. 293-297

[8] Yang Y, Lin Z and Liu F 2016 Stable imaging and accuracy issues of low-altitude unmanned aerial vehicle photogrammetry systems. Remote Sens. 8: 1-18

[9] Minaeian S, Liu J and Son Y J 2016 Vision-based target detection and localization via a team of cooperative UAV and UGVs. IEEE Trans. Syst. Man Cybern. Syst. 46: 1005-1016

[10] Meng W, He Z, Su R, Shehabinia A R, Lin L, Teo R and Xie L 2014 Decentralized control of multi-UAVs for target search, tasking and tracking. IFAC Proc. 47: 10048-10053
[11] Erdelj M, Natalizio E, Chowdhury K R and Akyildiz I F 2017 Help from the sky: Leveraging UAVs for disaster management. IEEE Pervasive Comput. 16: 24-32

[12] Aviram G and Rotman S R 2000 Analyzing the improving effect of modeled histogram enhancement on human target detection performance of infrared images. Infrared Phys. Technol. 41: 163-168

[13] Wang B J, Liu S Q, Li Q and Zhou H X 2006 A real-time contrast enhancement algorithm for infrared images based on plateau histogram. Infrared Phys. Technol. 48: 77-82

[14] Teo C K 2003 Digital enhancement of night vision and thermal images. Naval Postgraduate School Monterey CA.

[15] Branchitta F, Diani M, Corsini G and Romagnoli M 2009 New technique for the visualization of high dynamic range infrared images. Opt. Eng. 48: 096401-096409

[16] Lidong H, Wei Z, Jun W and Zebin S 2015 Combination of contrast limited adaptive histogram equalisation and discrete wavelet transform for image enhancement. IET Image Process. 9: 908-915

[17] Yang B S, Gu F and Ball A 2013 Thermal image enhancement using bi-dimensional empirical mode decomposition in combination with relevance vector machine for rotating machinery fault diagnosis. Mech. Syst. Signal Process. 38: 601-614

[18] Zuiderveld K 1994 Contrast limited adaptive histogram equalization. Graphics gems IV, San Diego, CA, USA, Academic Press Professional, Inc., pp. 474-485

[19] Wang X, Lin Z and Chen Z 2014 Augmented Lanczos bidiagonalization by small singular value decompositions for face recognition and image compression. Optik - Int. J. Light Electron Opt. 125: 4411-4416

[20] Makbol N M, Khoo B E and Rassem T H 2016 Block-based discrete wavelet transform-singular value decomposition image watermarking scheme using human visual system characteristics. IET Image Process. 10: 34-52

[21] Kumar A, Bhandari A K and Padhy P 2012 Improved normalised difference vegetation index method based on discrete cosine transform and singular value decomposition for satellite image processing. IET Signal Process. 6: 617-625

[22] Atta R and Abdel-Kader R F 2015 Brightness preserving based on singular value decomposition for image contrast enhancement. Optik - Int. J. Light Electron Opt. 126: 799-803

[23] Huang Z, Zhang T, Li Q and Fang H 2016 Adaptive gamma correction based on cumulative histogram for enhancing near-infrared images. Infrared Phys. Technol. 79: 205-215

[24] Yuan L T, Swee S K and Ping T C 2015 Infrared image enhancement using adaptive trilateral contrast enhancement. Pattern Recognit. Lett. 54: 103-108

[25] Wang Z, Bovik A C, Sheikh H R and Simoncelli E P 2004 Image quality assessment: from error visibility to structural similarity. IEEE Trans. Image Process. 13: 600-612

[26] Zhang L, Zhang L, Mou X and Zhang D 2011 FSIM: A feature similarity index for image quality assessment. IEEE Trans. Image Process. 20: 2378-2386 